\title{
Reseña: Comunidades. Estudios y experiencias sobre contextos y comunidades de aprendizaje
}

\section{Communities. Studies and experiences on contexts and learning communities}

\section{Comunidades. Estudos e experiências em contextos e comunidades de aprendizagem}

Martín, R. B., Rinaudo, Ma. C. y Paoloni, P. V. (Comp.) (2019). Comunidades. Estudios y experiencias sobre contextos y comunidades de aprendizaje. Villa María: Eduvim Editorial Universitaria Villa María. 208 p.

Daiana Schlegel

ORCID ID: 0000-0003-0583-5341

Universidad Nacional de Río Cuarto, Argentina

Autor referente: daiana-schlegel@hotmail.com

Historia editorial

Recibido: 10/12/2019 Aceptado: 01/03/2021

\section{RESUMEN}

Este trabajo, resume los contenidos del libro titulado Comunidades. Estudios y experiencias sobre contextos $y$ comunidades de aprendizaje (Martín, Rinaudo, \& Paoloni, 2019). Ofrece reflexiones personales acerca de una obra que, fundamentada en investigación científica, considera diferentes voces sobre comunidades de aprendizaje y práctica en diversos contextos educativos. El libro reseñado, presenta estudios enmarcados en enfoques 0 perspectivas socioculturales que entienden al aprendizaje como un proceso eminentemente social, que tiene lugar en las interacciones entre aprendices y las propiedades de ambientes específicos, independientemente del nivel de formalidad que se les haya adjudicado.

Palabras clave: Comunidades de aprendizaje; contextos; interacciones. 


\section{ABSTRACT}

This work summarizes the contents of the book entitled Communities. Studies and experiences on contexts and learning communities (Martín et al., 2019). It offers personal reflections about a work that, based on scientific research, considers different voices about learning and practice communities in different educational

contexts. The book reviewed presents studies framed in socio-cultural approaches or perspectives that understand learning as an eminently social process, which takes place in the interactions between learners and the properties of specific environments, regardless of the level of formality assigned to them.

Keywords: Learning communities; contexts; interactions.

\section{RESUMO}

Este trabalho resume o conteúdo do livro intitulado Comunidades. Estudos e experiências sobre contextos de aprendizagem e comunidades (Martín et al., 2019). Oferece reflexões pessoais sobre um trabalho que, a partir de pesquisas científicas, considera diferentes vozes sobre a aprendizagem e a prática das comunidades em diversos contextos educacionais. O livro, revisado, apresenta estudos enquadrados em abordagens socioculturais ou perspectivas que entendam a aprendizagem como um processo eminentemente social, que se dá nas interações entre aprendizes e nas propriedades de ambientes específicos, independentemente do nível de formalidade que lhes foi concedido.

Palavras-chave: Comunidades de aprendizagem; contextos; interações.

n los tiempos que corren, los contextos de aprendizaje no se reducen a la
educación formal, sino que van más allá de las aulas y sin límites de edad. Cada contexto es una configuración particular, una trama donde intervienen personas, objetos, contenidos, lugares, tareas y propósitos. Este entramado que se genera entre los componentes referidos, enriquece a cada uno de sus integrantes a partir de los conocimientos compartidos, de las experiencias vividas y de los intercambios generados. En este marco, las consideraciones en el campo de la educación se han desplazado hacia perspectivas más integrales. Como plantea Rinaudo (2014), en el libro que se reseña se entiende al "aprendiz" como protagonista en cada comunidad 
de aprendizaje o de práctica, donde cada participante se implica activamente en el análisis, comprensión y búsqueda de respuestas a las problemáticas emergentes, buscando la construcción de aprendizajes significativos por medio de experiencias que dejen "huella".

La obra aquí reseñada presenta un esbozo del desarrollo teórico que hay hasta el momento sobre contextos y comunidades de aprendizaje, tanto a nivel mundial, nacional, como local. Se suma a ello, el relato de experiencias reales que comparten las bases sobre una concepción del aprendizaje eminentemente social. Pero a su vez, las autoras proponen futuras líneas de investigación que abren nuevos interrogantes sobre las comunidades aprendizaje y de práctica en diversidad de contextos educativos. Se invita al lector a repensar y buscar el modo de abrir las puertas de la educación, "salir de las aulas" para acercarnos a los diferentes contextos que no solamente son promotores de diversidad de conocimientos, sino que también son tan ricos en sus resultados a partir de las redes que se generan entre sus participantes compartiendo nuevas experiencias y saberes.

El libro presenta trabajos de reflexión teórica y de experiencias de investigación, que se caracterizan por su rigor conceptual y metodológico. Está organizado en tres secciones, cuyo hilo conductor son los contextos y comunidades de aprendizaje, ampliando visiones al respecto y brindando herramientas para poder enriquecerlos. Tiene como eje transversal el interés por los problemas relativos a los modos en que actúa la cultura sobre el desarrollo y los aprendizajes humanos, desde una perspectiva plenamente sociocultural y explicaciones que enaltecen las vinculaciones entre éstos y sus escenarios culturales, históricos e institucionales.

La primera sección se titula "Estudios sobre contextos y comunidades de aprendizaje en el mundo", y realiza una presentación vinculada con desarrollos teóricos e investigaciones, referidos al tema eje del libro, a nivel mundial. El capítulo 1 se rotula Psicología Educacional: problemas persistentes, logros y metas a futuro, Rinaudo, 
(Argentina). En este capítulo, la autora -en base a una cuidadosa revisión bibliográficarealiza una reseña sobre el conocimiento en relación al campo de trabajo de la Psicología Educacional, ilustrando el modo en que los grandes investigadores, pertenecientes a ésta área de conocimiento, han tratado de contribuir con el logro de mayor y mejor educación para todos. El capítulo brinda un panorama actualizado, a nivel internacional, sobre las líneas de investigación en las áreas centrales del campo de estudio; sin embargo, evidencia la poca difusión de muchos hallazgos. Y en cuanto al contexto argentino, se detalla el modo en que las investigaciones a nivel internacional fueron consideradas -selección de las fuentes, enfoques y líneas de estudio, análisis de la evolución de los contenidos y enfoques del campo-. La Psicología Educacional en la actualidad se define como terreno multidisciplinario en el que aumenta el interés sobre estudios relativos a contextos de aprendizaje, pero continúan los problemas y enfoques de investigación que dejan fuera otros estudios de relevancia. El punto está en dar lugar a nuevas líneas de investigación.

El capítulo 2, se titula Comunidades de Aprendizagem e suas bases de transformação no Brasil, Rodrigues de Mello, Marini Braga y Coimbra Marigo, (Brasil) -. A partir del abordaje de una propuesta de transformación de escuelas en Comunidades de Aprendizaje en Brasil, las autoras ofrecen elementos teóricos y metodológicos de relevancia, poniendo al descubierto la articulación necesaria entre escuela y comunidad, focalizando en la función social de la escuela en la construcción de una sociedad mejor para todas las personas. En tal sentido, este capítulo describe la iniciativa de la Comunidad de Investigación de Excelencia para Todos (CREA), Universidad de Barcelona (CREA-UB / España) -con más de 35 años de antigüedaddonde se apunta a la transformación de las escuelas en Comunidades de Aprendizaje con el objetivo de promover una educación de calidad para todos (niños, jóvenes y personas adultas), en medio de la creación de interacciones más solidarias y respetuosas. En esta misma dirección, las autoras enuncian el proceso según el cual 
una escuela puede convertirse en Comunidad de Aprendizaje. Este proceso estaría conformado por dos etapas interrelacionadas: de entrada y de consolidación. La etapa de entrada supone a su vez una sucesión de momentos -conciencia, toma de decisiones, sueños, selección de prioridades, planificación-. La etapa de consolidación, integra las fases de investigación, formación y evaluación. Toda transformación de escuelas en comunidades de aprendizaje yace sobre las necesidades de los contextos educativos, que son producto del diálogo.

El capítulo 3 se titula Narrativas, Enseñanza y Universidad, Leite Méndez, Rivas Flores y Cortes González, (España), y presenta diversas experiencias de trabajos con narrativas en la enseñanza universitaria -tanto en el grado como en el posgrado- de sujetos sobre la experiencia escolar en su formación como docentes. Estas tramas articulan componentes sociales, personales, políticos, culturales, ideológicos e históricos, recuperando a los sujetos y a partir de allí pensando en un diálogo crítico con otras narrativas que pueden abrir espacios de transformación, de re-creación, superando la reproducción. Entre las experiencias se enuncia el trabajo con biografías escolares, relatos subyacentes de las performances, las redes sociales como narrativas, narrativas poéticas, musicales y artísticas, relatos simultáneos del profesorado, como así también el diálogo entre narrativas. El trabajo expone, con una sólida revisión bibliográfica, el porqué del trabajo de las narrativas en la universidad, y es enriquecido por algunas experiencias, cambios educativos emergentes del trabajo colaborativo y de la comprensión de la experiencia vivida, situada y colectiva. Ofrece nuevas representaciones, desde una perspectiva centrada en el sujeto, a partir de la articulación de relatos, miradas y de la interacción con diversos colectivos y contextos, pensando y reflexionando sobre el sentido de la enseñanza en la universidad para abrir otras vías.

La segunda sección del libro se denomina Estudios sobre comunidades de práctica y aprendizaje en Argentina. En esta ocasión, la autora -Rocío B. Martín-, focaliza en el 
análisis en líneas de trabajo relacionadas con las comunidades de aprendizaje y de práctica en Argentina, sintetizando su trabajo de tesis doctoral. La sección se organiza en dos capítulos: el capítulo 4 y 5 , respectivamente. El capítulo 4 se denomina Perspectiva teórica sobre el estudio de los contextos y comunidades de aprendizaje, y deja muy en claro qué se entiende por contextos, su diversidad, y la posibilidad del aprendizaje en comunidad, la participación y el aprendizaje situado en la práctica. De manera más precisa, en primer lugar, se describen estudios del aprendizaje desde la teoría situada, cognición situada y aprendizaje situado; luego, se presentan avances conceptuales referidos a comunidades de práctica en función a tres líneas de investigación, antecedentes y con definiciones conceptuales sobre aprendizaje y las características o dimensiones de las comunidades de aprendizaje como contextos promisorios para el aprendizaje. Por último, se define Contextos de Aprendizaje, particularizando los criterios para su delimitación, diferentes tipos de contextos educativos, características de los mismos, y haciendo foco en los contextos no formales de aprendizaje, se enuncian rasgos comunes, como así también, la clasificación de acuerdo a sus posibles funciones.

El capítulo 5 se titula Tres estudios sobre comunidades de aprendizaje y práctica en contextos no formales. Con un amplio marco de referencia, el capítulo presenta los resultados de tres estudios - tal como lo anticipa el título -, los cuales fueron Taller de Tejido, Curso profesional de formación de guardavidas y Asociación de Diabetes, orientados a analizar las características de las comunidades de aprendizaje, describiendo actividades e interacciones, trayectorias, rol de las emociones y significados que se les asignan a las experiencias. Particularmente, relata en detalle en cada uno de los estudios una breve contextualización, los objetivos que lo guiaban, aspectos metodológicos, materiales y modalidad de recolección de datos, procedimientos, análisis y resultados, acompañados de evidencia empírica. Finaliza con una síntesis de los tres estudios, organizándolos en cuatro temas: comunidades 
de aprendizaje y de práctica, la forma de los contextos no formales, emociones y participación en contexto. Los resultados obtenidos, las categorías de análisis propuestas y las interpretaciones elaboradas, son originales y constituyen un aporte novedoso al entendimiento de las comunidades de aprendizaje y práctica, al interior de cada experiencia en particular, como así también del análisis articulado de los diferentes casos. Por ejemplo, se destaca la oportunidad que este capítulo brinda para repensar las bases teóricas de las comunidades de aprendizaje y de práctica para diseñar contextos poderosos para el aprendizaje, considerando sus diferentes formas y atendiendo a la participación de sus miembros, su implicación, como así también la dinámica emocional entre ellos.

Finalmente, la tercera y última sección, denominada Lineamientos para futuras investigaciones sobre comunidades de aprendizaje y de práctica en diversidad de contextos educativos, está compuesta por el capítulo 6, titulado Senderos promisorios para futura investigación sobre comunidades de aprendizaje y de práctica, escrito por Martín et al., 2019. En este capítulo, se sistematizan los desarrollos presentados en el libro y delimitan, de modo fundamentado, orientaciones generales para futuras investigaciones. Particularmente, presentan cinco líneas de estudio promisorias para el surgimiento y desarrollo de comunidades educativas, destacando la posibilidad de que en la práctica investigativa se entrelacen y enriquezcan mutuamente. Esta posibilidad, incrementaría así el alcance en su conjunto de cada una de estas propuestas presentadas.

En síntesis, como puede apreciarse, la obra, cumple con la meta propuesta, la cual es, "brindar aportes teóricos y empíricos que amplíen y enriquezcan la perspectiva sobre el estudio de las comunidades y su potencial para promover procesos de enseñanza y de aprendizaje óptimos en diversos contextos educativos" (Martín et al., 2019, p. 200). De ésta manera nos aporta herramientas para comprender, repensar y cuestionar los procesos de enseñanza y de aprendizaje en sí mismos como modo posible de avanzar 
hacia contribuciones capaces de promover mejoras educativas que se traduzcan en sociedades más democráticas, justas y comprometidas con el bien común. La importancia de contribuir al surgimiento de comunidades de aprendizaje en diversos contextos educativos se arraiga en la idea de promover aprendizajes con sentido donde primen los procesos de co-construcción; es decir, entornos que den lugar a un aprendizaje fundamentalmente colaborativo, donde la participación, la implicancia genuina y el compromiso sostenido de cada uno de sus integrantes, se conviertan en rasgos identitarios y en donde las distancias socio-emocionales entre los integrantes se acorten y tracen senderos de actuación hacia metas compartidas.

\section{Referencias}

Martín, R. B., Rinaudo, M. C., \& Paoloni, P. V. (2019). Comunidades. Estudios y experiencias sobre contextos y comunidades de aprendizaje. Villa María: Eduvim - Editorial Universidad Villa María.

Rinaudo, M. C. (2014). Estudios sobre los contextos de aprendizaje. Arenas y Fronteras. En P. V. Paoloni, M. C. Rinaudo, \& A. González Fernández, Cuestiones en Psicología Educacional: perspectivas teóricas, metodológicas y estudios de campo. (págs. 163-206). Tenerife: Sociedad Latinoamericana de Comunicación Social (SLCS).

\section{Declaración de contribución de autores}

DS contribuyó con la totalidad del artículo.

\section{Editor se sección}

La editora de sección de este artículo fue Mónica Da Silva.

ORCID ID: 0000-0003-2522-4716 
Formato de citación

Schlegel, D. (2021). Reseña: Comunidades. Estudios y experiencias sobre contextos y comunidades de aprendizaje. Psicología, Conocimiento y Sociedad, 11(1), 244-252. doi: http://dx.doi.org/10.26864/PCS.v11.n1.11 